\title{
Invasive lobular carcinoma of the breast: morphology, biomarkers and 'omics
}

\author{
Amy E McCart Reed ${ }^{1 \dagger}$, Jamie R Kutasovic ${ }^{1 \dagger}$, Sunil R Lakhani ${ }^{1,2,3}$ and Peter T Simpson ${ }^{1,3^{*}}$
}

\begin{abstract}
Invasive lobular carcinoma of the breast is the most common 'special' morphological subtype of breast cancer, comprising up to $15 \%$ of all cases. Tumours are generally of a good prognostic phenotype, being low histological grade and low mitotic index, hormone receptor positive and HER2, p53 and basal marker negative, and with a generally good response to endocrine therapy. Despite this, clinicians face countless challenges in the diagnosis and long-term management of patients, as they encounter a tumour that can be difficult to detect through screening, elicits a very invasive nature, a propensity for widespread metastatic colonisation and, consequently, in some studies a worse long-term poor outcome compared with invasive carcinoma of no special type. Here we review the morphological and molecular features that underpin the disparate biological and clinical characteristics of this fascinating tumour type.
\end{abstract}

\section{Introduction}

Invasive lobular carcinoma (ILC) is the most common 'special' type of breast cancer and presents with a distinct morphology and clinical behaviour compared with invasive carcinoma of no special type (IC-NST). Typically, ILC tumours display features associated with a good prognosis, being low grade and oestrogen receptor positive; however, the tumour can be highly metastatic [1] and several studies demonstrate that the overall longterm outcome for patients diagnosed with ILC may be similar or worse than for patients diagnosed with IC-NST $[2,3]$. E-cadherin loss is responsible for the inherently

\footnotetext{
* Correspondence: p.simpson@uq.edu.au

${ }^{\dagger}$ Equal contributors

'The University of Queensland, UQ Centre for Clinical Research, Herston, QLD 4029, Australia

${ }^{3}$ School of Medicine, The University of Queensland, Herston, QLD 4006, Australia

Full list of author information is available at the end of the article
}

discohesive phenotype associated with ILCs, and changes at the genomic level account for this loss. Recent technological advances have generated masses of genomic and transcriptomic data, some of which is further illuminating the natural history of ILCs. We present here a review of lobular carcinoma, paying particular attention to the morphological and immunophenotypic features of pre-invasive and invasive lesions, the importance of E-cadherin dysfunction in tumour biology, transcriptomics, genomics and diagnostic aspects that aid patient management.

\section{Morphological characteristics of lobular neoplasia and invasive lobular carcinoma}

Diagnostic criteria for lobular neoplasia (LN) and ILC (Figure 1) are now well established and described [4] and so are only briefly outlined below. The term 'lobular neoplasia' was introduced [5] to encompass a spectrum of in situ neoplastic proliferations including atypical lobular hyperplasia (ALH) and lobular carcinoma in situ (LCIS), which describe different levels of involvement of individual lobular units. The descriptions ALH and LCIS are widely used to classify these lesions since they confer different relative risks (4- to 5 -fold and 8- to 10 -fold, respectively) for the patient to subsequently develop invasive cancer compared with the general population [6]. By definition, neoplastic cells of LN remain confined to the terminal duct-lobular unit, but they may exhibit pagetoid spread in which cells can migrate along the ductal system between the basement membrane and normal epithelial cell population (Figure 2).

The cells of LN and ILC are typically small, monomorphic and lack cohesion, with round or notched ovoid nuclei and a thin rim of cytoplasm. Intra-cytoplasmic lumen, containing a central inclusion of mucin, may be present and in some cells this may be large enough to create a signet ring cell-type appearance (Figure 1). Cells of classic LCIS or ILC may vary in appearance and have been referred to as type A cells (classic) or the larger type B cells (vesicular nuclei) that may show mild pleomorphism. Cells of the pleomorphic type of LCIS (PLCIS) 

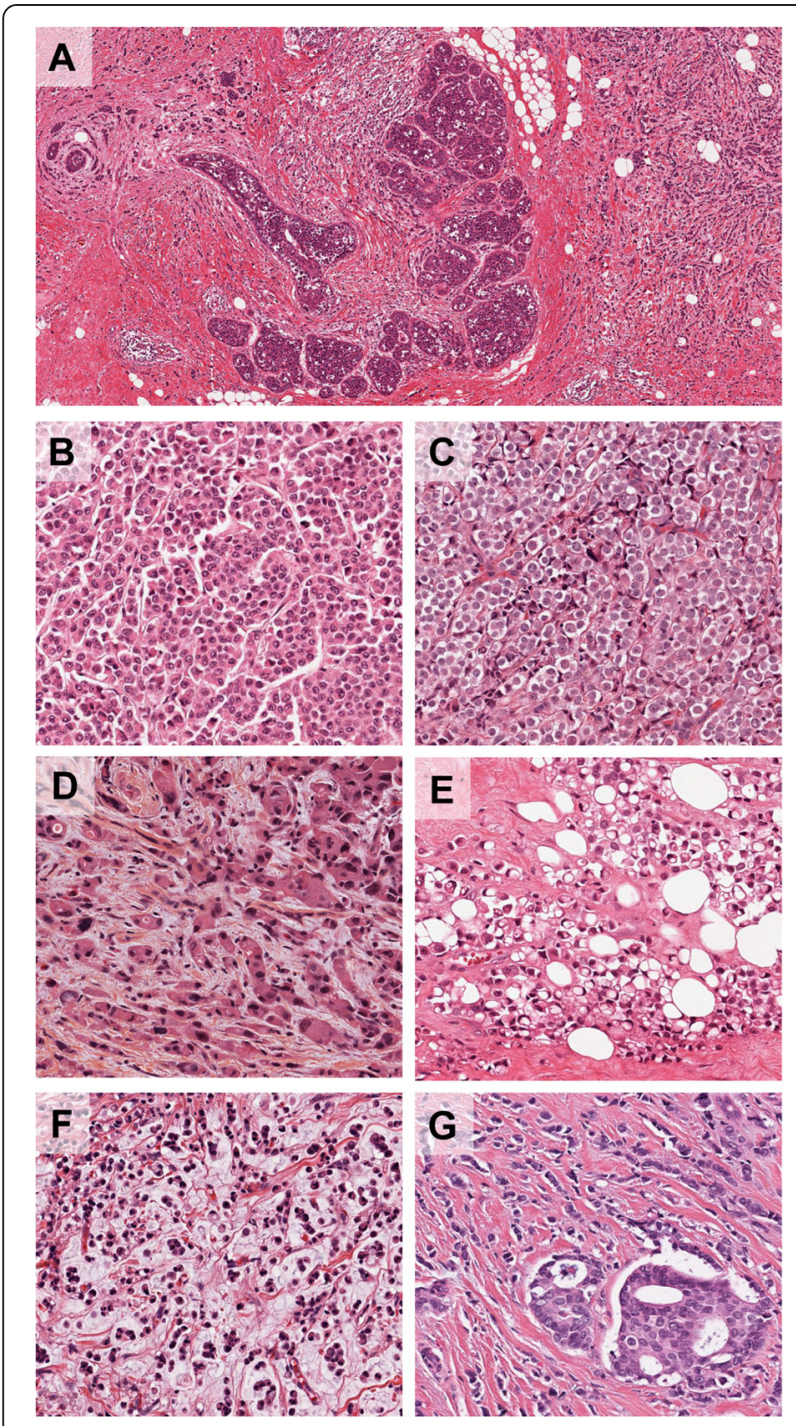

Figure 1 Morphological characteristics of invasive lobular carcinoma and its variants. (A) Low power view of a terminal duct lobular unit colonised by lobular carcinoma in situ. Classic invasive lobular carcinoma is seen diffusely infiltrating the whole specimen as single cells and single files of cells. The characteristic targetoid growth pattern is evident on the left-hand side (see also Figure 2). (B-G) Morphological variants of the classic type: (B) alveolar type, with globular aggregates of approximately 20 cells; (C) solid type with discohesive tumour cells growing in solid sheets; (D) a pleomorphic

variant - note the pink, foamy cytoplasm typical of an apocrine phenotype and irregular nuclei; (E) pleomorphic invasive lobular carcinoma with prominent signet ring cells; $(\mathbf{F})$ invasive lobular carcinoma showing mucinous/histiocytoid morphology; (G) mixed ductal-lobular carcinoma.

may be larger still and exhibit marked nuclear pleomorphism, akin to that observed in high-grade ductal carcinoma in situ (DCIS) [7]. Extensive or florid LCIS is also important to recognize. These lesions are characterized by the proliferation of the same type A or type B cells, but there is marked expansion of involved lobular units and areas of necrosis and microcalcification [8].

In classical ILC, the characteristic pattern of growth involves the infiltration of single cells or single files of cells through the stroma, with little disturbance of normal tissue architecture. The invading tumour cells are frequently arranged in a concentric (targetoid) pattern around normal ducts or structures (Figure 2). There are a series of morphologically recognised variants that demonstrate either cytological or architectural variation of the characteristic features of classic ILC. Pleomorphic lobular carcinoma (PLC) retains the distinctive growth pattern of classic ILC but, as in its in situ counterpart (PLCIS), there is marked cellular atypia and nuclear pleomorphism relative to classic LN and ILC. PLC may also have an increased mitotic rate, be composed of signet ring cells (Figure 1) and/or show apocrine or histiocytoid differentiation. Conversely, the solid and alveolar variants are both characterized by classic ILC cells (small, regular sized and lacking cohesion) that are arranged in sheets (solid type) or in aggregates of at least 20 cells (alveolar type, Figure 1) rather than in single cords of cells. Solid ILC may also be more frequently pleomorphic and mitotically active relative to classic ILC. Classic ILC may be admixed with one or more of these morphological variants or with tumour cells of a tubular growth pattern (tubulo-lobular carcinoma). Furthermore, around $5 \%$ of all invasive breast tumours exhibit mixed features of both ductal and lobular differentiation $[4,9]$ (Figure 1).

Histological grading is an important part of breast tumour classification, and is performed using the Nottingham histological grading system. There is debate, however, as to the relevance of this system for the 'special types' including lobular carcinomas and some studies suggest it is of limited value since tubule formation is rare (except in the tubulo-lobular variant), there is limited nuclear pleomorphism and the mitotic count is frequently low. Consequently, most ILCs, including variants, are grade 2. Nevertheless, other studies report that grade is indeed an independent prognostic factor in ILC, as it is in breast cancer in general, with mitotic count being the most useful component for predicting poor outcome [10]. Furthermore, while several studies report that morphological variants are aggressive subtypes associated with poor outcome, particularly relative to classic type [11], evidence suggests that a nuclear pleomorphism score of 3 (which would indicate a classification of PLC), in an overall grade 2 tumour does not add prognostic value, the most important discriminator being overall grade and/or mitotic count [12].

ALH, LCIS and PLCIS can be frequently found colocalised in the same specimen, also alongside other non-obligate precursors such as columnar cell lesions, 

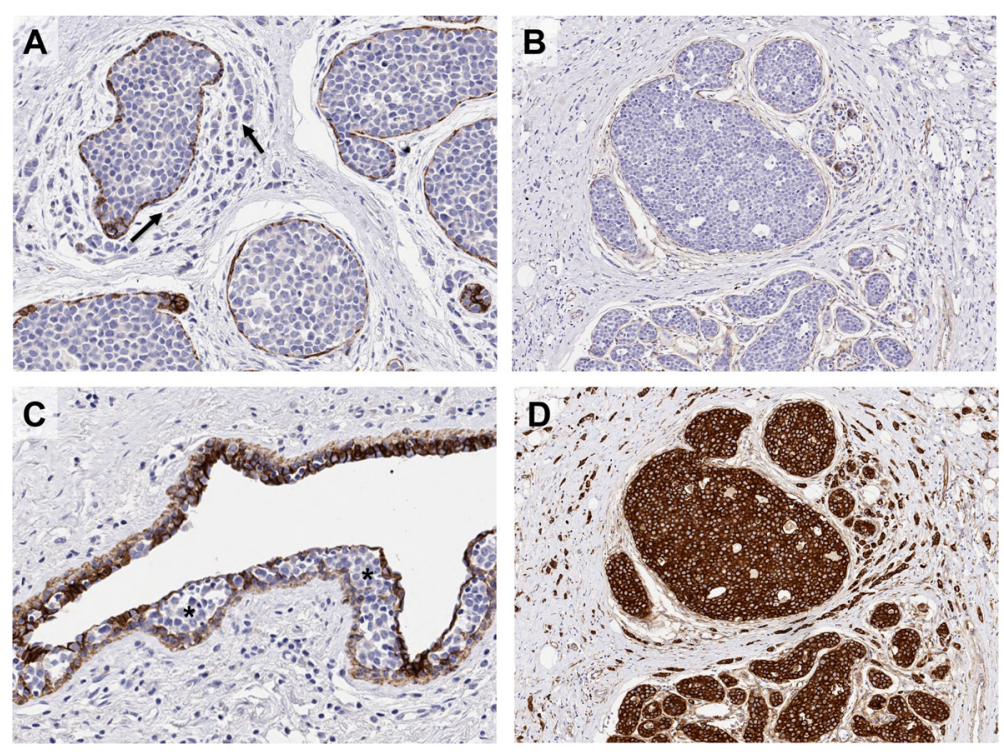

Figure $\mathbf{2}$ Immunohistochemical staining of E-cadherin and its binding complex in invasive lobular carcinoma. Lobular carcinoma in situ (LCIS) and invasive lobular carcinoma (ILC); note invasive neoplastic cells of ILC (arrows) growing in a targetoid fashion around the in situ component. (A) E-cadherin and (B) $\beta$-catenin staining is negative in both LCIS and ILC, although positive staining is observed in the myoepithelial cells surrounding the LCIS. (C) Pagetoid spread (asterisks) is also observed in this case, whereby the neoplastic cells (negative for E-cadherin) are growing and invading between the luminal and myoepithelial cells of a normal ductal structure). (D) In the absence of E-cadherin, there is a strong re-localisation of p120-catenin to the cytoplasm of neoplastic cells in LCIS and ILC.

atypical ductal hyperplasia and low-grade DCIS as part of the 'low-grade' family of breast precursor lesions [13]. LNs frequently co-exist with invasive carcinomas of lobular type, including classic ILC (Figures 1 and 2) and tubulo-lobular carcinomas (in $90 \%$ and $57 \%$ of cases, respectively [13]), supporting a common evolutionary origin of these lesions. Indeed, the overlapping cytological appearance and frequent co-localisation of LN and ILC, combined with concordant immunophenotypic and molecular characteristics, supports the notion that LCIS and PLCIS are clonal and non-obligate precursor lesions for ILC and PLC, respectively $[14,15]$.

\section{Immunophenotyping invasive lobular carcinoma}

Classic ILCs are almost always hormonally regulated. Up to $95 \%$ of cases express oestrogen receptor (ER) $\alpha$ and 60 to $70 \%$ of cases express progesterone receptor $[2,16,17]$, whereas only 60 to $70 \%$ of IC-NST express these two biomarkers. ER $\alpha$ is always expressed in the alveolar variant $(100 \%)$ yet is less frequently found in pleomorphic ILC (10 to $76 \%)[10,18]$. The androgen receptor and ER $\beta$ are also expressed in approximately $90 \%$ of ILCs $[10,19]$. The interplay between these receptors in ILC is yet to be fully elucidated, though it is clear the high frequency of hormone receptor expression reflects the overall good response to endocrine-based therapies [2].

Biomarkers associated with poor clinical behaviour are rarely expressed in ILC, including the HER2, p53 and basal/myoepithelial markers (cytokeratins 14 and 5/6, epidermal growth factor receptor, smooth muscle actin and p63) $[10,16,17]$. Generally the proliferation index (measured by Ki67 staining) is low in ILC, reflecting the low mitotic count (see above) and this likely contributes to reduced response to chemotherapy relative to patients diagnosed with IC-NST. Pleomorphic ILCs, on the other hand, are more likely to exhibit HER2 amplification (in 35 to $80 \%$ of cases) and p53 expression and the proliferation index is typically higher $[10,18]$.

\section{E-cadherin dysfunction - master regulator of the lobular phenotype}

The characteristic discohesive growth pattern of ILC is the result of the dysregulation of cell-cell adhesion properties, primarily driven by the targeted disruption of the cell adhesion molecule E-cadherin. E-cadherin is a calciumdependent transmembrane protein that mediates cell-cell adhesion and cellular polarity by binding to itself on neighbouring cells in a homophilic manner. The intracellular domain of E-cadherin associates with the actin cytoskeleton via $\alpha-, \beta-, \gamma-$ and $p 120$ catenins to form adherens junctions between non-neural epithelial cells. E-cadherin is largely regulated by its catenin-binding partners, which anchor E-cadherin to the membrane and the actin cytoskeleton. E-cadherin-mediated cell adhesion maintains cell viability and when this adhesion is lost the detached cells undergo a cell death program called anoikis. 
In normal breast epithelial cells and in most IC-NST, $\mathrm{E}$-cadherin and the associated catenin binding proteins are located at the cell membrane, maintaining cellular cohesion. In contrast, approximately $90 \%$ of LNs and ILCs, including variants, completely lack E-cadherin protein expression [15,20-23]. The loss of E-cadherin in ILC also results in the loss of $\alpha$-, $\beta$ - and $\gamma$-catenins, and p120-catenin becomes up-regulated and re-localised to the cytoplasm [24]. From a biological point of view, this re-localisation of p120 has been found to enable anoikis resistance in lobular cells, allowing them to survive independently of attachment to neighbouring epithelial cells and promote cell migration through activation of Rho/ Rock signalling [25]. E-cadherin expression has become an important diagnostic feature of LN and ILC and the cytoplasmic localisation of p120-catenin is a positive immunohistochemistry marker for ILC [26]. In combination these biomarkers may aid in classification when it is difficult to differentiate between lobular and ductal lesions [26]; however, there remains confusion regarding the interpretation and so caution is warranted. In particular it is important to remember that approximately $10 \%$ of ILCs still express E-cadherin [10,22], either with normal membrane localisation or aberrantly distributed as fragmented membrane and/or cytoplasmic staining. The E-cadherin-catenin complex may be dysfunctional in these cases due to the presence of $C D H 1$ gene mutation (see below) or aberrant/loss of expression of the catenin binding proteins [22], which may be detected using $\beta$-catenin and p120-catenin immunohistochemistry. However, a diagnosis of LN or ILC based on morphologic and cytologic criteria should therefore not be reclassified as DCIS or IC-NST based on the status of these immunohistochemical markers [26].

E-cadherin deregulation occurs in the earliest morphological stage of lobular tumourigenesis (that is, ALH) and is frequently and irreversibly driven by genomic alterations targeting its gene, $\mathrm{CDH1}$ (located at chromosome 16q22.1). Molecularly, the patterns of E-cadherin loss often follow Knudsen's two hit hypothesis for a classic tumour suppressor gene, involving CDH1 mutation, gene methylation and/or loss of heterozygosity in the region of 16q22.1 (frequently involving the whole chromosomal arm).

Promoter hypermethylation and concomitant downregulation of $C D H 1$ expression has been reported in 21 to $77 \%$ of ILCs $[27,28]$ and the detection of methylated $C D H 1$ promoter sequences in adjacent normal tissues and in LN implies that this is an early hit [29]. The somatic copy number loss of 16q in ILC and ER-positive, low-grade IC-NST is extremely frequent, suggesting these tumours share a common pathway of evolution. We reviewed the DNA copy number status at the CDH1 gene locus in the 153 lobular tumours from The Cancer
Genome Atlas (TCGA) data resource [30,31] and this revealed that $12.4 \%$ of tumours show a diploid copy number; $84.3 \%$ show a single copy loss and $3.3 \%$ show a putative homozygous deletion. Chromosomal analysis of LNs has shown they too lose chromosome 16q [8,32-34], suggesting this is also an early assault on the $C D H 1$ gene region.

Somatic mutations are found dispersed throughout the $\mathrm{CDH1}$ coding region and are frequently truncating [21] (Figure 3). Identical $C D H 1$ genetic mutations have been detected in LCIS and in their adjacent invasive counterpart [15], which is a key finding implicating LCIS as a direct (but non-obligate) precursor for ILC. Further to this, CDH1 mutations were detected in LCIS [35], although, surprisingly, no mutations were found in adjacent, microdissected ALH lesions. This may be a question of technological sensitivity and so the application of highresolution massively parallel sequencing technologies is certainly warranted to clarify such findings.

The reported frequency of $C D H 1$ mutation and loss of heterozygosity is unexpectedly discrepant between studies (from 30 to $80 \%$ ) [10,21,36]. Improving technologies and increasing cohort sizes have not necessarily resolved this. For example, TCGA [37] reported from an exome sequencing strategy (that is, enriching for exons only) that $C D H 1$ mutations were very common $(30 / 36$; 83\%) within the lobular histological subtype and, expectedly, corresponded with low E-cadherin expression. TCGA resource has now made comprehensive 'omic data available for 958 breast cancers through the cBioPortal $[30,31]$ and in an investigation of these data we identified CDH1 mutations in 78 out of 155 ILCs (50\%). This latter figure is supported by an independent exome sequencing study of ER-positive tumours in the clinical context of aromatase inhibitor response, where they identified CDH1 mutation in 20 out of 40 ILCs [38].

Mutations in $C D H 1$ have also been identified in other types of epithelial cancers, most notably in diffuse gastric carcinomas, which have a very similar infiltrative growth pattern to ILC of the breast. Hereditary diffuse gastric carcinoma is sometimes caused by a germline mutation in $C D H 1$ [39] and mutation carriers have an increased risk for developing ILC. A diagnosis of ILC may also be enriched within breast cancer families and since LNs/ ILCs more frequently present as multifocal or bilateral disease, it fits with a theory of a germline predisposition to tumour development. Despite E-cadherin being the obvious candidate for such a predisposition, early work suggested $C D H 1$ germline variants are rare in familial lobular breast cancer [40] but do account for some cases of bilateral ILC [41]. Considerable evidence arising from studying the human disease therefore exists for E-cadherin playing a major role in the initiation and biology of both lobular and diffuse gastric cancer. Animal 


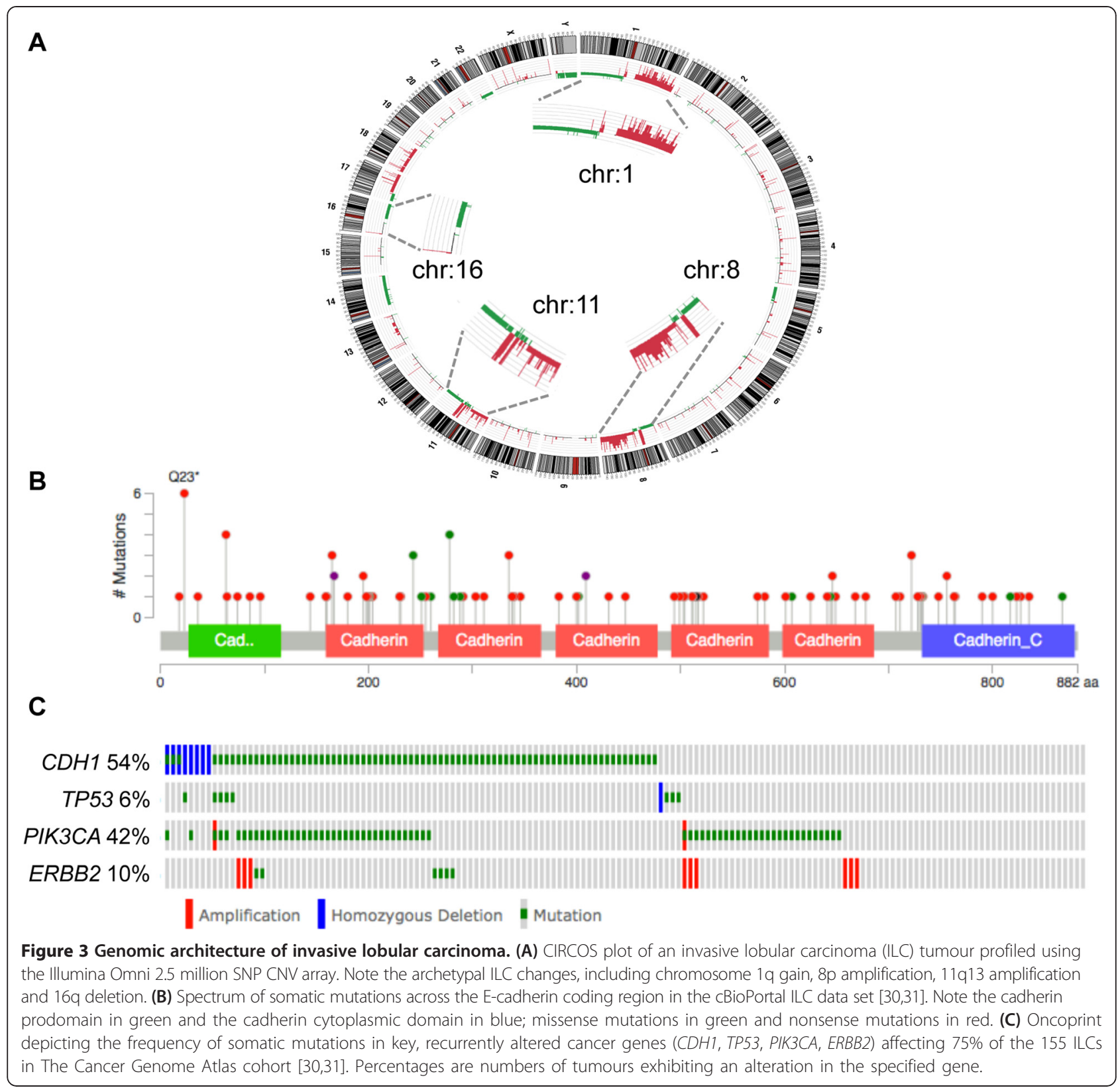

models of hereditary diffuse gastric cancer and lobular breast cancer provide additional support for this concept, whereby $C D H 1$ germline deficiency in combination with a second hit (carcinogen treatment or TP53 mutation) is sufficient to initiate disease development $[42,43]$. (These aspects are covered in more detail in a review in this series [44].)

The loss of E-cadherin is also associated with the process of epithelial to mesenchymal transition (EMT) where cells lose polarity and adhesion to become more migratory and invasive during embryonic morphogenesis and wound healing. Tumour cells are believed to be able to hijack this process to facilitate migration away from the primary tumour microenvironment and metastatic dissemination. The acquisition of the mesenchymal phenotype is accompanied by cadherin switching (loss of E-cadherin and activation of $\mathrm{N}$-cadherin), which is driven by transcriptional regulators of E-cadherin, including SNAIL and TWIST, as well as post-transcriptionally active microRNAs (for example, the miR200 family), and the gain in expression of mesenchymal markers, such as vimentin. Given the loss of E-cadherin and the infiltrating growth pattern of ILC, it is tempting to speculate that EMT plays a mechanistic role in driving this phenotype. Indeed, a meta-analysis of microarray gene expression data found TWIST to be highly expressed in human ILC 
samples, showing 70\% had elevated TWIST mRNA expression, compared with $32 \%$ of ductal carcinomas [45]. However, immunohistochemical analysis of EMT markers in human breast tumours demonstrated that: i) neoplastic lobular cells retain their epithelial identity; ii) TWIST protein was expressed by the fibroblasts in the prominent stromal component of ILC; and iii) only 1 out of 24 (4\%) ILCs expressed EMT markers [46]. While EMT is traditionally associated with late stages of tumour progression (invasion and metastasis) and is a dynamic process, the loss of E-cadherin in ILC is an early and typically irreversible event in ILC. Thus, the functional role of EMT in driving the invasive nature of ILC remains unlikely.

\section{Transcriptome profiling of lobular tumours}

At the turn of the century, a pivotal study used gene expression profiling microarrays to categorise breast cancers into a series of 'intrinsic' subtypes that stratified prognosis: luminal A, luminal B, HER2, and basal-like $[47,48]$. These categories have since been expanded to include claudin-low $[47,48]$ and normal breast-like. Due to the nature of ILCs generally being low-grade and ERpositive, they are frequently classed as luminal $\mathrm{A}$, and owing to their commonly infiltrative histology and thus a comparatively reduced tumour to stroma cellularity (compared to ductal tumours), they may also be classed as normal-like, simply as a consequence of there being more normal cells and/or stroma in their processed samples [49]. Ultimately though, like ductal carcinomas, they are a heterogeneous group and have the potential to be classed as any of the defined subtypes, including molecular apocrine for the PLC variant [48,50], while, interestingly, the non-lobular special types of breast cancer (for example, medullary, metaplastic, micropapillary, tubular, apocrine and neuroendocrine carcinomas) cluster within a single subtype only, underscoring their more inherent homogeneity.

Gene expression profiling studies have also been undertaken to better understand the biological differences between lobular and ductal invasive tumours. Overall, the number of lobular tumours profiled has been considerably lower than that for ductal invasive tumours [51-55]). Korkola and colleagues [52] defined 11 genes as capable of differentiating ILCs from ductal carcinomas, but more recent studies report larger, functional groups of genes as being responsible for their different aetiologies. Of most relevance are those functional gene groups that were identified when 20 ILCs were compared with 91 ER-positive, grade-matched invasive ductal carcinomas (IDCs): adhesion, transforming growth factor beta signalling; cell communication and trafficking; actin remodelling; lipid/prostaglandin synthesis; transcription factor/immediate early genes [54]. Ultimately, other than expected transcriptional changes associated with E-cadherin dysfunction, at the individual transcript level there was minimal overlap between all five studies. Given the variety of platforms used for these assays, small sample sizes and modes of analysis, this is not altogether surprising. A meta-analysis of these studies identified THBS4 (thrombospondin 4) as a potential ILC biomarker, but investigations at the protein level confirmed no difference in expression between ILCs and their ductal counterparts, and instead revealed THBS4 as a marker of tumour-associated extracellular matrix [56]. Again, this finding is probably more associated with the fact that ILC tumours exhibit a higher stromal content, thus skewing the subsequent downstream analyses.

\section{The genomic landscape of lobular carcinomas}

LNs and ILCs are more likely to be diploid than ductal tumours [16]. Indeed, chromosomal and array-based comparative genomic hybridisation (aCGH) analyses have defined, on a gross scale, the genomic profile of lobular carcinomas - in short, they harbour fewer chromosomal changes than ductal carcinomas and are generally less complex. Genomic losses, such as at $16 \mathrm{p}$, $16 q, 17 p$ and $22 q$, and gains at $6 q$ were detected in $\mathrm{LN}$ by chromosomal CGH [33]. The key alterations identified more recently by aCGH in classic LCIS, florid/ extensive LCIS and PLCIS are 1q gain and 16q loss, with increased genomic complexity observed in the latter two groups of lesions, including loss of $8 \mathrm{p}, 11 \mathrm{q}$ and $17 \mathrm{p}$ and amplifications at 11q13 (CCND1) and 17q12 (ERBB2) $[8,14,34]$. Like their pre-invasive counterparts and ERpositive IC-NST, both classic and pleomorphic ILC exhibit a high frequency of gain of chromosome 1q and loss of $16 q[18,23,57,58]$ and it has been reported that all ILCs lose at least part of 16q [58]. Other recurrent alterations include losses at 8p23-p21, 11q14.1-q25, and 13q, gains of $8 \mathrm{q}$ and 16p, and high-level amplifications at 1q32, 8p12p11.2, and 11q13. Although some candidate genes in the various regions have been postulated (for example, FGFR1 in 8p12-p11.2 and CCND1 in 11q13 [23]), no definitive data confirming the drivers contained in these various regions have been reported specifically for lobular breast cancer. This is likely a result of the complexity of the chromosomal changes and the context-dependent nature of some of these alterations. Numerous candidate oncogenes have been identified in these regions but not specifically for lobular tumours - for example, ZNF703 gene amplification at $8 \mathrm{p} 12$ specifies luminal B breast cancer [59]. As mentioned above, PLC contains a similar profile of chromosomal change, although there is increased complexity and additional amplifications are present - 8q24 (MYC), 17q12 (ERBB2/Her2) and 20q13, which are usually considered to be archetypal changes of high-grade ductal tumours [18]. Some attempts have been made to classify tumour genome profiles based on genomic 
architecture as either simple, complex-firestorm or complex-sawtooth. The genomes of both classic and pleomorphic ILC are generally classified as simple (in that they frequently harbour 1q gain and 16q loss and few other alterations) or complex-firestorm (relating to the additional presence of complex, high-level amplifications at the stated loci) [18,23]. It is conceivable that those ILCs that are classed as complex-firestorm have a worse prognosis, though this has yet to be explored.

A catalogue of the transcriptomic and genomic architecture of 2,000 breast cancers, and their integration into novel clusters was reported in 2012 [60]. The discovery set of this large study included 148 classic ILCs, of which $88.5 \%$ were ER positive and were classified as: luminal A, 44.9\%; luminal B, 19.7\%; basal, 2.7\%; HER2, 6.1\%; normal, $25.9 \%$. This study also presented an alternative categorisation algorithm combining transcriptome and genomic copy number data to define 10 'integrative clusters' (IntClusts). ILCs were predominantly assigned to IntClust 3 (39.7\%), 4 (23.5\%) and 8 (14.7\%), with varying frequencies of the archetypal chromosomal changes $(1 \mathrm{q}+, 16 \mathrm{p}+, 16 \mathrm{q}-)$. Predictably, IntClust 3 , into which most ILCs segregated, showed overall the simplest genomes, a high frequency of $1 \mathrm{q}+$ and $16 \mathrm{q}-$ and the best survival outcome. Similarly, tumours in IntClust 8 also harbour a high frequency of $1 \mathrm{q}+$ and $16 \mathrm{q}-$, but also $16 \mathrm{p}+$. Conversely, tumours in IntClust 4 showed infrequent $1 \mathrm{q}+$ and $16 \mathrm{q}-$. The groups in which lobular carcinomas are not enriched (that is, less than approximately $6 \%$ of the ILCs) showed more recurrent gains/ amplifications on chromosomes 8q, 11q or 17q. Subtle variation in the genomic alterations in these tumours may therefore have a strong influence on tumour behaviour.

\section{The data era: 'next-generation' sequencing}

Significant technological advances in recent years have meant that the depth of interrogation of individual tumour genomes has increased significantly. This socalled 'next-generation sequencing' technology combined with the activities of several large consortia has led to the production of masses of high quality sequence and genomic copy number data for large numbers of tumours. As noted above, two studies have performed exome sequencing on ILC of any significant numbers $[37,38]$. Beyond the highly recurrent mutations in $C D H 1$ and PIK3CA, which for PIK3CA the mutation rate is similar to that observed overall in ER-positive breast cancers, there is a paucity of recurrent driver mutations in this tumour type (Table 1), supporting the idea that heterogeneity within and between tumours is complex.

One of the first studies to report the application of the then novel sequencing technologies to breast cancer samples was that of Shah and colleagues in 2009 [61].
Table 1 Recurrent mutations in invasive lobular carcinoma

\begin{tabular}{llll}
\hline Gene & $\begin{array}{l}\mathbf{c B i o P o r t a l}[\mathbf{3 0 , 3 1 ]} \\
(\mathbf{n}=\mathbf{1 5 5})\end{array}$ & $\begin{array}{l}\text { Ellis et al. [38] } \\
(\mathbf{n}=\sim 40)^{\mathbf{a}}\end{array}$ & $\begin{array}{l}\text { Ross et al. [62] } \\
(\mathbf{n}=\mathbf{2 2})^{\mathbf{b}}\end{array}$ \\
\hline AKT1 & $0.6 \%$ & $\mathrm{NR}$ & $9.1 \%$ \\
ARID1A & $4.5 \%$ & $\mathrm{NR}$ & $\mathrm{NA}$ \\
ATR & $2.0 \%$ & $2.5 \%$ & $\mathrm{NA}$ \\
BIRC6 & $2.0 \%$ & $2.5 \%$ & $\mathrm{NA}$ \\
CDH1 & $50.0 \%$ & $50.0 \%$ & $100 \%$ \\
CDKN1B & $1.3 \%$ & $3.4 \%$ & $\mathrm{NA}$ \\
ERBB2 & $3.8 \%$ & $\mathrm{NR}$ & $18 \%$ \\
GATA3 & $2.6 \%$ & $3.4 \%$ & $\mathrm{NA}$ \\
KRAS & $0.6 \%$ & $\mathrm{NR}$ & $9.1 \%$ \\
MALAT1 & $0.0 \%$ & $2.5 \%$ & $\mathrm{NA}$ \\
MAP2K4 & $0.6 \%$ & $3.4 \%$ & $9.1 \%$ \\
MAP3K1 & $5.0 \%$ & $13.8 \%$ & $\mathrm{NA}$ \\
NCOR1 & $4.5 \%$ & $9.1 \%$ & $\mathrm{NA}$ \\
NF1 & $3.2 \%$ & $\mathrm{NR}$ & $\mathrm{NA}$ \\
PIK3CA & $41.0 \%$ & $41.4 \%$ & $36.4 \%$ \\
RB1 & $1.30 \%$ & 0 & $9.1 \%$ \\
RUNX1 & $7.0 \%$ & $5.0 \%$ & $4.5 \%$ \\
SMAD4 & $1.30 \%$ & $\mathrm{NR}$ & $\mathrm{NA}$ \\
TP53 & $5.0 \%$ & $12.1 \%$ & $36.4 \%$ \\
\hline & & &
\end{tabular}

${ }^{a}$ The exact number of invasive lobular carcinomas in the Ellis study is difficult to define as several cohorts were assessed using a variety of technologies. ${ }^{\mathrm{b}}$ The Ross study selected only $\mathrm{CDH} 1$ mutant cases. NA, not available; NR, not reported.

This study sequenced a pleural effusion metastasis and matched primary ILC diagnosed 9 years earlier and found that 5 somatic mutations (of a possible 32 defined for the metastasis) were present in the primary tumour, a telling comment on the degree of clonal evolution occurring during progression from primary clone to metastasis. This patient also had an ERBB2 mutation, as did 2 of 192 ILCs in their validation set. Somatic mutations (not including amplifications) in ERBB2 have since been shown to be generally rare in breast cancer but interestingly were significantly enriched in the ILC subtype [37]. Consulting the cBioPortal $[30,31]$ for an updated data review, 6 of 155 ILCs (3.9\%) harboured an ERBB2 mutation. Interestingly, in a massively parallel, targeted amplicon sequencing of 'actionable cancer genes' in ILC post-treatment relapse (that is, recurrence or metastasis), Ross and colleagues [62] reported HER2/ERBB2 genetic alterations in 6 of 22 (27\%) cases, including 4 mutations, one gene fusion and one amplification. HER2 is an important clinically actionable target, indicating this type of targeted sequencing analysis, which has greater sensitivity than exome sequencing and is applicable to formalin-fixed paraffin-embedded tissue, may soon aid in the management of patients when planning primary or secondary treatment regimes. 


\section{Diagnostic algorithms}

As the era of molecular technology for subtyping disease and identifying molecular targets takes huge leaps forward it is tempting to begin to ignore the more traditional morphological classification of disease and consider molecular subtyping (for example, luminal, basal, HER2 subtypes) and testing (for example, OncotypeDX) for classification and management. However, the breast cancer morphological special types remain fundamental to the management of patients since the biological and clinical significance of these entities provides important information regarding appropriate management strategies and outcomes.

A diagnosis of lobular carcinoma, as a special morphological type, supports this idea, since there are clinical and biological features that set it apart from the more commonly diagnosed IC-NST, and despite the good prognostic features' exhibited by ILC, some large studies consistently demonstrate that ILCs have a similar or worse long-term outcome compared with IC-NST $[2,3]$. Many of the challenging aspects in the diagnosis and management of ILC relate to the indolent but highly infiltrative nature of the tumour growth and the biology of dysfunctional E-cadherin that underpins this. For instance, LNs and ILCs are not always detected as a palpable mass and can be difficult to detect by mammography [63] owing to the rare association with calcification and the lack of host stromal response to the diffusely infiltrating tumour.

Differentiating classic LCIS from its morphological variants (that is, extensive/florid LCIS and PLCIS) may be important from a management point of view, owing to anecdotal evidence that these lesions have a different clinical course and that they exhibit more genomic instability $[8,14,34]$. Correct diagnostic classification of LN is also very important because the management of patients diagnosed with $\mathrm{LN}$ are different to those with DCIS, in the setting of core needle biopsy or surgical margin status, where further excision is required for all cases of DCIS but not for LN. There is a significant body of literature regarding this and readers are directed to $[10,26,64]$ and references therein for more information. Briefly, differentiating LCIS and PLCIS from low- and high-grade DCIS, respectively, or lesions with indeterminate features may be difficult in certain scenarios. The use of ancillary immunohistochemical staining for E-cadherin, $\beta$-catenin and p120-catenin can therefore be helpful to aid classification $[24,26]$. In terms of ILC, histological grading is considered a critical component of classification, and description of the morphological variants is recommended given the prognostic insight this may provide and the potential for future epidemiological and biological studies related to tumour subtyping $[11,12,65]$. As above, the use of E-cadherin, p120 catenin or $\beta$-catenin is appropriate to help resolve the diagnosis of difficult cases, although it is important to consider classification first based on morphology and cytology and not to reclassify a bona fide ILC as IC-NST based on 'normal' E-cadherin or p120-catenin staining since around $10 \%$ of ILCs still express membranous E-cadherin [20,22,26]. Pan-cytokeratin markers are also utilized to differentiate small ILC cells from macrophages in biopsies and extremely diffuse cases.

ILCs respond less well to chemotherapy compared to IC-NST, probably resulting in part from their indolent, low proliferative index (low mitotic count and low Ki-67 index). Many molecular tests are now available to prognosticate and inform decisions regarding the addition of chemotherapy to a patient's treatment program. Many ILC tumours meet the requirements for the Oncotype DX 21-gene clinical assay, in that they are generally grade 2 and ER positive, and may not have spread to the lymph nodes. The usefulness of this and other tests is reviewed in [66], where it is also noted that many of these signatures focus on proliferation as a mechanism of assessing likelihood of recurrence.

Expression of ER, progesterone receptor and HER2 guide therapeutic decisions and the vast majority of patients will receive endocrine-based therapy, for which there is generally a good response [2]; however, de novo or acquired resistance is an inevitable problem for some patients. The somatic mutation profile of a tumour may contribute to this; for example, tumours harbouring or acquiring driver mutations in ESR1 [67] or ERBB2 [37] or amplifications at $8 \mathrm{p} 12$ (FGFR1) or $11 \mathrm{q} 13$ (CCND1) [23] may be less responsive to targeted endocrine therapy. In support of this, the ER-positive ILC cell line model MDA-MB-134VI was found to be de novo tamoxifen resistant, yet cells were sensitized to anti-oestrogen therapy when in combination with FGFR1 inhibitors [68]. Oestrogen-related receptor gamma/AP1 signalling may also mediate tamoxifen resistance in the SUM44 cell model system [69]. Recent research has also shown that PIK3CA mutations are selected for during progression from the primary ILC tumour to a local recurrence but not through to dissemination of distant metastases [70]. While links between PIK3CA mutation and endocrine therapy resistance have been investigated in some breast cancers, this mechanism has not been specifically studied in ILC; however, it is reasonable to hypothesize that this may be the case in some endocrine-resistant ILCs [71]. A gene expression study comparing ILC and IDC tumour biopsies in the neo-adjuvant setting suggests that letrozole both induces near identical transcriptome changes in the tumour types and does not interfere with histological subtype-specific gene expression [72]. Recent data suggest there may be an improved response to the aromatase inhibitor letrozole compared with tamoxifen in ILCs but the 
biological mechanisms driving the differences in response need to be further investigated [73]. As our understanding of the biological mechanisms that underpin response and resistance to anti-oestrogen therapies improves we will be able to better predict which treatment regime would be most effective (endocrine therapy or in combination with other targeted therapies).

\section{Conclusion}

Lobular carcinoma is an important breast cancer subtype with some peculiar clinical and biological characteristics compared with the more commonly diagnosed IC-NST. Rather surprisingly, and despite the good prognostic features of the primary tumour and good response to endocrine therapy, the long-term outcome for patients diagnosed with ILC is, in some studies, worse than for IC-NST. There remain significant challenges, therefore, managing patients with this specific disease. Although considered a 'special' histological type, the disease is heterogeneous, and so identifying patients with poor prognostic subtypes will likely provide benefit in delineating more personalized and aggressive treatment or monitoring for disease progression. A detailed assessment of the genomic landscape of a large cohort of ILCs with long-term follow-up and/or in the context of treatment resistance will no doubt be essential to moving forward with precision medicine for patients diagnosed with this tumour type.

\section{Note}

This article is part of a series on Lobular breast cancer, edited by Ulrich Lehmann. Other articles in this series can be found at http://breast-cancer-research.com/series/LBC.

\section{Abbreviations \\ aCGH: Array-based comparative genomic hybridisation; ALH: Atypical lobular hyperplasia; CGH: Comparative genomic hybridisation; DCIS: Ductal carcinoma in situ; EMT: Epithelial to mesenchymal transition; ER: Oestrogen receptor; IC-NST: Invasive carcinoma no special type; IDC: Invasive ductal carcinoma; ILC: Invasive lobular carcinoma; IntClust: Integrative cluster; LCIS: Lobular carcinoma in situ; LN: Lobular neoplasia; PLC: Pleomorphic lobular carcinoma; PLCIS: Pleomorphic lobular carcinoma in situ; TCGA: The Cancer Genome Atlas.}

\section{Competing interests}

The authors declare that they have no competing interests.

\begin{abstract}
Acknowledgements
We apologise to authors whose work we were not able to discuss due to space limitations. We thank Sarah Sim for insightful discussion. PTS was the recipient of a fellowship from the National Breast Cancer Foundation, Australia.
\end{abstract}

\section{Author details}

'The University of Queensland, UQ Centre for Clinical Research, Herston, QLD 4029, Australia. 'Pathology Queensland, The Royal Brisbane \& Women's and Gold Coast Hospitals, Herston, QLD 4029, Australia. ${ }^{3}$ School of Medicine, The University of Queensland, Herston, QLD 4006, Australia.

Published online: 30 January 2015

\section{References}

1. Ferlicot S, Vincent-Salomon A, Medioni J, Genin P, Rosty C, Sigal-Zafrani B, et al. Wide metastatic spreading in infiltrating lobular carcinoma of the breast. Eur J Cancer. 2004:40:336-41.

2. Rakha EA, El-Sayed ME, Powe DG, Green AR, Habashy H, Grainge MJ, et al. Invasive lobular carcinoma of the breast: response to hormonal therapy and outcomes. Eur J Cancer. 2008;44:73-83.

3. Pestalozzi BC, Zahrieh D, Mallon E, Gusterson BA, Price KN, Gelber RD, et al. Distinct clinical and prognostic features of infiltrating lobular carcinoma of the breast: combined results of 15 International Breast Cancer Study Group clinical trials. J Clin Oncol. 2008;26:3006-14.

4. Lakhani SR, Ellis IO, Schnitt SJ, Tan PH, van de Vijver MJ. WHO Classification of Tumours of the Breast. 4th ed. Lyon: IARC; 2012.

5. Haagensen CD, Lane N, Lattes R, Bodian C. Lobular neoplasia (so-called lobular carcinoma in situ) of the breast. Cancer. 1978;42:737-69.

6. Page DL, Kidd Jr TE, Dupont WD, Simpson JF, Rogers LW. Lobular neoplasia of the breast: higher risk for subsequent invasive cancer predicted by more extensive disease. Hum Pathol. 1991;22:1232-9.

7. Sneige N, Wang J, Baker BA, Krishnamurthy S, Middleton LP. Clinical, histopathologic, and biologic features of pleomorphic lobular (ductal-lobular) carcinoma in situ of the breast: a report of 24 cases. Mod Pathol. 2002;15:1044-50.

8. Shin SJ, Lal A, De Vries S, Suzuki J, Roy R, Hwang ES, et al. Florid lobular carcinoma in situ: molecular profiling and comparison to classic lobular carcinoma in situ and pleomorphic lobular carcinoma in situ. Hum Pathol. 2013;44:1998-2009.

9. Rakha EA, Gill MS, El-Sayed ME, Khan MM, Hodi Z, Blamey RW, et al. The biological and clinical characteristics of breast carcinoma with mixed ductal and lobular morphology. Breast Cancer Res Treat. 2009;114:243-50.

10. Rakha EA, Ellis IO. Lobular breast carcinoma and its variants. Semin Diagn Pathol. 2010;27:49-61.

11. Iorfida M, Maiorano E, Orvieto E, Maisonneuve P, Bottiglieri L, Rotmensz N, et al. Invasive lobular breast cancer: subtypes and outcome. Breast Cancer Res Treat. 2012;133:713-23.

12. Rakha EA, van Deurzen $\mathrm{CH}$, Paish EC, Macmillan RD, Ellis IO, Lee AH, Pleomorphic lobular carcinoma of the breast: is it a prognostically significant pathological subtype independent of histological grade? Mod Pathol. 2013:26:496-501.

13. Abdel-Fatah TM, Powe DG, Hodi Z, Lee AH, Reis-Filho JS, Ellis IO. High frequency of coexistence of columnar cell lesions, lobular neoplasia, and low grade ductal carcinoma in situ with invasive tubular carcinoma and invasive lobular carcinoma. Am J Surg Pathol. 2007;31:417-26.

14. Reis-Filho JS, Simpson PT, Jones C, Steele D, Mackay A, Iravani M, et al. Pleomorphic lobular carcinoma of the breast: role of comprehensive molecular pathology in characterization of an entity. J Pathol. 2005;207:1-13.

15. Vos CB, Cleton-Jansen AM, Berx G, de Leeuw WJ, ter Haar NT, van Roy F, et al. E-cadherin inactivation in lobular carcinoma in situ of the breast: an early event in tumorigenesis. Br J Cancer. 1997;76:1131-3.

16. Arpino G, Bardou VJ, Clark GM, Elledge RM. Infiltrating lobular carcinoma of the breast: tumor characteristics and clinical outcome. Breast Cancer Res. 2004;6:R149-56

17. Sastre-Garau X, Jouve M, Asselain B, Vincent-Salomon A, Beuzeboc P, Dorval T, et al. Infiltrating lobular carcinoma of the breast. Clinicopathologic analysis of 975 cases with reference to data on conservative therapy and metastatic patterns. Cancer. 1996;77:113-20.

18. Simpson PT, Reis-Filho JS, Lambros MB, Jones C, Steele D, Mackay A, et al. Molecular profiling pleomorphic lobular carcinomas of the breast: evidence for a common molecular genetic pathway with classic lobular carcinomas. J Pathol. 2008:215:231-44.

19. Skliris GP, Munot K, Bell SM, Carder PJ, Lane S, Horgan K, et al. Reduced expression of oestrogen receptor beta in invasive breast cancer and its re-expression using DNA methyl transferase inhibitors in a cell line model. J Pathol. 2003;201:213-20.

20. Rakha EA, Patel A, Powe DG, Benhasouna A, Green AR, Lambros MB, et al. Clinical and biological significance of E-cadherin protein expression in invasive lobular carcinoma of the breast. Am J Surg Pathol. 2010;34:1472-9.

21. Berx G, Cleton-Jansen AM, Strumane K, de Leeuw WJ, Nollet F, van Roy F, et al. E-cadherin is inactivated in a majority of invasive human lobular breast cancers by truncation mutations throughout its extracellular domain. Oncogene. 1996;13:1919-25. 
22. Da Silva L, Parry S, Reid L, Keith P, Waddell N, Kossai M, et al. Aberrant expression of E-cadherin in lobular carcinomas of the breast. Am J Surg Pathol. 2008;32:773-83

23. Reis-Filho JS, Simpson PT, Turner NC, Lambros MB, Jones C, Mackay A, et al. FGFR1 emerges as a potential therapeutic target for lobular breast carcinomas. Clin Cancer Res. 2006;12:6652-62.

24. Dabbs DJ, Bhargava R, Chivukula M. Lobular versus ductal breast neoplasms: the diagnostic utility of p120 catenin. Am J Surg Pathol. 2007;31:427-37

25. Schackmann RC, van Amersfoort M, Haarhuis JH, Vlug EJ, Halim VA, Roodhart JM, et al. Cytosolic p120-catenin regulates growth of metastatic lobular carcinoma through Rock1-mediated anoikis resistance. J Clin Invest. 2011;121:3176-88.

26. Dabbs DJ, Schnitt SJ, Geyer FC, Weigelt B, Baehner FL, Decker T, et al. Lobular neoplasia of the breast revisited with emphasis on the role of E-cadherin immunohistochemistry. Am J Surg Pathol. 2013;37:e1-11.

27. Droufakou S, Deshmane V, Roylance R, Hanby A, Tomlinson I, Hart IR. Multiple ways of silencing E-cadherin gene expression in lobular carcinoma of the breast. Int J Cancer. 2001:92:404-8.

28. Sarrio D, Moreno-Bueno G, Hardisson D, Sanchez-Estevez C, Guo M, Herman JG, et al. Epigenetic and genetic alterations of APC and $\mathrm{CDH} 1$ genes in lobular breast cancer: relationships with abnormal E-cadherin and catenin expression and microsatellite instability. Int J Cancer. 2003;106:208-15.

29. Zou D, Yoon HS, Perez D, Weeks RJ, Guilford P, Humar B. Epigenetic silencing in non-neoplastic epithelia identifies E-cadherin (CDH1) as a target for chemoprevention of lobular neoplasia. J Pathol. 2009:218:265-72.

30. Cerami E, Gao J, Dogrusoz U, Gross BE, Sumer SO, Aksoy BA, et al. The cBio cancer genomics portal: an open platform for exploring multidimensional cancer genomics data. Cancer Discov. 2012;2:401-4.

31. Gao J, Aksoy BA, Dogrusoz U, Dresdner G, Gross B, Sumer SO, et al. Integrative analysis of complex cancer genomics and clinical profiles using the cBioPortal. Sci Signal. 2013;6:pl1

32. Simpson PT, Reis-Filho JS, Gale T, Lakhani SR. Molecular evolution of breast cancer. J Pathol. 2005;205:248-54.

33. Lu YJ, Osin P, Lakhani SR, Di Palma S, Gusterson BA, Shipley JM. Comparative genomic hybridization analysis of lobular carcinoma in situ and atypical lobular hyperplasia and potential roles for gains and losses of genetic material in breast neoplasia. Cancer Res. 1998;58:4721-7.

34. Chen YY, Hwang ES, Roy R, DeVries S, Anderson J, Wa C, et al. Genetic and phenotypic characteristics of pleomorphic lobular carcinoma in situ of the breast. Am J Surg Pathol. 2009:33:1683-94

35. Mastracci TL, Tjan S, Bane AL, O'Malley FP, Andrulis IL. E-cadherin alterations in atypical lobular hyperplasia and lobular carcinoma in situ of the breast. Mod Pathol. 2005:18:741-51.

36. Cleton-Jansen AM. E-cadherin and loss of heterozygosity at chromosome 16 in breast carcinogenesis: different genetic pathways in ductal and lobular breast cancer? Breast Cancer Res. 2002;4:5-8.

37. Network CGA. Comprehensive molecular portraits of human breast tumours. Nature. 2012;490:61-70.

38. Ellis MJ, Ding L, Shen D, Luo J, Suman VJ, Wallis JW, et al. Whole-genome analysis informs breast cancer response to aromatase inhibition. Nature. 2012:486:353-60

39. Guilford P, Hopkins J, Harraway J, McLeod M, McLeod N, Harawira P, et al. E-cadherin germline mutations in familial gastric cancer. Nature. 1998:392:402-5.

40. Schrader KA, Masciari S, Boyd N, Salamanca C, Senz J, Saunders DN, et al. Germline mutations in $\mathrm{CDH} 1$ are infrequent in women with early-onset or familial lobular breast cancers. J Med Genet. 2011;48:64-8.

41. Petridis C, Shinomiya I, Kohut K, Gorman P, Caneppele M, Shah V, et al. Germline $\mathrm{CDH} 1$ mutations in bilateral lobular carcinoma in situ. $\mathrm{Br} J$ Cancer. 2014;110:1053-7.

42. Derksen PW, Liu X, Saridin F, van der Gulden H, Zevenhoven J, Evers B, et al. Somatic inactivation of E-cadherin and p53 in mice leads to metastatic lobular mammary carcinoma through induction of anoikis resistance and angiogenesis. Cancer Cell. 2006;10:437-49.

43. Humar B, Blair V, Charlton A, More H, Martin I, Guilford P. E-cadherin deficiency initiates gastric signet-ring cell carcinoma in mice and man. Cancer Res. 2009;69:2050-6.

44. Christgen M, Derksen PW. Lobular breast cancer: molecular basis, mouse and cellular models. Breast Cancer Res. in press.
45. Yang J, Mani SA, Donaher JL, Ramaswamy S, Itzykson RA, Come C, et al. Twist, a master regulator of morphogenesis, plays an essential role in tumor metastasis. Cell. 2004;117:927-39.

46. Sarrio D, Rodriguez-Pinilla SM, Hardisson D, Cano A, Moreno-Bueno G, Palacios J. Epithelial-mesenchymal transition in breast cancer relates to the basal-like phenotype. Cancer Res. 2008:68:989-97.

47. Perou CM, Sorlie T, Eisen MB, van de Rijn M, Jeffrey SS, Rees CA, et al. Molecular portraits of human breast tumours. Nature. 2000;406:747-52.

48. Prat A, Parker JS, Karginova O, Fan C, Livasy C, Herschkowitz Jl, et al. Phenotypic and molecular characterization of the claudin-low intrinsic subtype of breast cancer. Breast Cancer Res. 2010;12:R68.

49. Mackay A, Weigelt B, Grigoriadis A, Kreike B, Natrajan R, A'Hern R, et al. Microarray-based class discovery for molecular classification of breast cancer: analysis of interobserver agreement. J Natl Cancer Inst. 2011;103:662-73.

50. Weigelt B, Horlings HM, Kreike B, Hayes MM, Hauptmann M, Wessels LF, et al. Refinement of breast cancer classification by molecular characterization of histological special types. J Pathol. 2008;216:141-50.

51. Gruel N, Lucchesi C, Raynal V, Rodrigues MJ, Pierron G, Goudefroye R, et al. Lobular invasive carcinoma of the breast is a molecular entity distinct from luminal invasive ductal carcinoma. Eur J Cancer. 2010;46:2399-407.

52. Korkola JE, DeVries S, Fridlyand J, Hwang ES, Estep AL, Chen YY, et al. Differentiation of lobular versus ductal breast carcinomas by expression microarray analysis. Cancer Res. 2003;63:7167-75.

53. Turashvili G, Bouchal J, Baumforth K, Wei W, Dziechciarkova M, Ehrmann J, et al. Novel markers for differentiation of lobular and ductal invasive breast carcinomas by laser microdissection and microarray analysis. BMC Cancer. 2007:7:55.

54. Weigelt B, Geyer FC, Natrajan R, Lopez-Garcia MA, Ahmad AS, Savage K, et al. The molecular underpinning of lobular histological growth pattern: a genome-wide transcriptomic analysis of invasive lobular carcinomas and grade- and molecular subtype-matched invasive ductal carcinomas of no special type. J Pathol. 2010;220:45-57.

55. Zhao H, Langerod A, Ji Y, Nowels KW, Nesland JM, Tibshirani R, et al. Different gene expression patterns in invasive lobular and ductal carcinomas of the breast. Mol Biol Cell. 2004;15:2523-36.

56. McCart Reed AE, Song S, Kutasovic JR, Reid LE, Valle JM, Vargas AC, et al. Thrombospondin-4 expression is activated during the stromal response to invasive breast cancer. Virchows Arch. 2013;463:535-45.

57. Loo LW, Grove DI, Williams EM, Neal CL, Cousens LA, Schubert EL, et al. Array comparative genomic hybridization analysis of genomic alterations in breast cancer subtypes. Cancer Res. 2004;64:8541-9.

58. Roylance R, Gorman P, Papior T, Wan YL, Ives M, Watson JE, et al. A comprehensive study of chromosome $16 \mathrm{q}$ in invasive ductal and lobular breast carcinoma using array CGH. Oncogene. 2006;25:6544-53.

59. Sircoulomb F, Nicolas N, Ferrari A, Finetti P, Bekhouche I, Rousselet E, et al. ZNF703 gene amplification at 8p12 specifies luminal B breast cancer. EMBO Mol Med. 2011:3:153-66.

60. Curtis C, Shah SP, Chin SF, Turashvili G, Rueda OM, Dunning MJ, et al. The genomic and transcriptomic architecture of 2,000 breast tumours reveals novel subgroups. Nature. 2012;486:346-52.

61. Shah SP, Morin RD, Khattra J, Prentice L, Pugh T, Burleigh A, et al. Mutational evolution in a lobular breast tumour profiled at single nucleotide resolution. Nature. 2009:461:809-13.

62. Ross JS, Wang K, Sheehan CE, Boguniewicz AB, Otto G, Downing SR, et al. Relapsed classic E-cadherin (CDH1)-mutated invasive lobular breast cancer shows a high frequency of HER2 (ERBB2) gene mutations. Clin Cancer Res. 2013;19:2668-76.

63. Hilleren DJ, Andersson IT, Lindholm K, Linnell FS. Invasive lobular carcinoma: mammographic findings in a 10-year experience. Radiology. 1991;178:149-54.

64. Niell B, Specht M, Gerade B, Rafferty E. Is excisional biopsy required after a breast core biopsy yields lobular neoplasia? AJR Am J Roentgenol. 2012;199:929-35.

65. Rakha EA, El-Sayed ME, Menon S, Green AR, Lee AH, Ellis IO. Histologic grading is an independent prognostic factor in invasive lobular carcinoma of the breast. Breast Cancer Res Treat. 2008;111:121-7.

66. Gluz O, Hofmann D, Wurstlein R, Liedtke C, Nitz U, Harbeck N. Genomic Profiling in Luminal Breast Cancer. Breast Care (Basel). 2013;8:414-22.

67. Jeselsohn R, Yelensky R, Buchwalter G, Frampton G, Meric-Bernstam F, Gonzalez-Angulo AM, et al. Emergence of constitutively active estrogen receptor-alpha mutations in pretreated advanced estrogen receptor-positive breast cancer. Clin Cancer Res. 2014;20:1757-67. 
68. Sikora MJ, Cooper KL, Bahreini A, Luthra S, Wang G, Chandran UR, et al. Invasive lobular carcinoma cell lines are characterized by unique estrogenmediated gene expression patterns and altered tamoxifen response. Cancer Res. 2014;74:1463-74.

69. Riggins RB, Lan JP, Zhu Y, Klimach U, Zwart A, Cavalli LR, et al. ERRgamma mediates tamoxifen resistance in novel models of invasive lobular breast cancer. Cancer Res. 2008;68:8908-17.

70. Christgen M, Noskowicz M, Schipper E, Christgen H, Heil C, Krech T, et al. Oncogenic PIK3CA mutations in lobular breast cancer progression. Genes Chromosomes Cancer. 2013;52:69-80.

71. Miller TW, Balko JM, Arteaga CL. Phosphatidylinositol 3-kinase and antiestrogen resistance in breast cancer. J Clin Oncol. 2011;29:4452-61.

72. Arthur LM, Turnbull AK, Webber VL, Larionov AA, Renshaw L, Kay C, et al. Molecular changes in lobular breast cancers in response to endocrine therapy. Cancer Res. 2014;74:5371-6.

73. Metzger O, Giobbie-Hurder A, Mallon E, Viale G, Winer E, Thürlimann B, et al. Relative effectiveness of letrozole compared with tamoxifen for patients with lobular carcinoma in the BIG 1-98 trial [abstract]. In: Thirty-Fifth Annual CTRC-AACR San Antonio Breast Cancer Symposium, vol. 72. San Antonio, TX Cancer Research; 2012. p. S1-1. 